\title{
Diseño de un sistema de suministro de energía para vehículos eléctricos usando lógica difusa
}

\author{
Ismael Osuna Galán ${ }^{1}$, Yolanda Pérez Pimentel ${ }^{1}$, Juan Villegas Cortez ${ }^{2}$, \\ Carlos Avilés Cruz ${ }^{2}$ \\ ${ }^{1}$ Universidad Politécnica de Chiapas, \\ México \\ ${ }^{2}$ Universidad Autónoma Metropolitana Azcapotzalco, \\ México
}

\{iosuna,ypimentel\}@upchiapas.edu.mx, \{juanvc, caviles\}@ correo.azc.uam.mx

Resumen. La tecnología de los sistemas electrónicos de potencia se ha diversificado en áreas industriales, comerciales y residenciales. Actualmente, los carros eléctricos usan diversos controladores que les permiten tener una eficiencia comparable con los carros convencionales. Los vehículos eléctricos (EV) son sistemas mecatrónicos complejos descritos por modelos no lineales y, por lo tanto, su diseño y análisis de control no es una tarea fácil. De igual forma, incrementar el rendimiento de un EV no depende de crear mejores sistemas de almacenamiento. Este artículo muestra el desarrollo de un sistema de gestión energética basado en lógica difusa para un vehículo eléctrico con la finalidad de minimizar su consumo total de energía y optimizar el banco de baterías. Los resultados experimentales usando el controlador difuso se comparan con los resultados en condiciones normales de operación. Se observa un incremento en el rendimiento de las baterías y del rendimiento en general del consumo de energía. Las señales de velocidad adquiridas muestran una mejora en algunos parámetros dinámicos, tales como el sobreimpulso, el tiempo de establecimiento y el error de estado estable. Se muestra que este controlador difuso aumenta la eficiencia energética general del vehículo.

Palabras clave: lógica difusa, vehículos eléctricos, control de energía.

\section{Design of a Power Supply System for Electric Vehicles using Fuzzy Logic}

\begin{abstract}
The technology of electronic power systems has diversified into industrial, commercial and residential areas. Currently, electric cars use various controllers that allow them to have an efficiency comparable to conventional cars. Electric vehicles (EV) are complex mechatronic systems described by nonlinear models and, therefore, their design and control analysis is not an easy task. Similarly, increasing the performance of an EV does not depend on creating better storage systems. This article shows the development of an energy management system based on fuzzy logic for an electric vehicle in order to minimize its total energy consumption and optimize the battery bank. The
\end{abstract}


experimental results using the fuzzy controller are compared with the results under normal operating conditions. There is an increase in the performance of the batteries and the overall performance of the energy consumption. Acquired velocity signals show an improvement in some dynamic parameters, such as overshoot, set-up time and steady-state error. It is shown that this fuzzy controller increases the overall energy efficiency of the vehicle.

Keywords: fuzzy logic, electric vehicles, energy control.

\section{Introducción}

Con las regulaciones de emisiones contaminantes, los avances en la tecnología de motores eléctricos y la creación de baterías de alto desempeño, los fabricantes de automóviles alrededor del mundo han comenzado a considerar más seriamente la comercialización de vehículos que usen fuentes de energía alternativas.

La tecnología aplicada a los vehículos eléctricos (EV) ha logrado un desempeño comparable con los motores de combustión interna, dado que los motores de tracción eléctrica proporcionan una aceleración rápida y el motor de combustión interna funciona bien a velocidades constantes es que se han creado los vehículos híbridos $[6,7]$.

Hoy en día, la gran mayoría de los vehículos eléctricos en el mercado usan una única fuente de energía basada en baterías para generar el funcionamiento del vehículo. La baja densidad de potencia y el corto ciclo de vida es una de las deficiencias de la batería electroquímica. La aparición de sistemas de almacenamiento de energía compuesto tales como celdas solares, baterías de alto rendimiento, sistemas electrónicos de potencia son grandes avances.

El uso de tecnología de electrónica de potencia junto con un sistema que controle el consumo de energía del banco de baterías reduce el ciclo de descarga de corriente de las baterías y aumenta la distancia de desplazamiento del EV. [8]

Este artículo propone un enfoque para la administración de energía en aplicaciones en el EV UPChis01 (Fig. 1) basado en la reducción de la corriente de potencia aplicada al banco de baterias. Una de las principales ventajas de esta propuesta es la introducción de un sistema difuso capaz de mejorar la vida útil del banco de baterías y mejorar su rendimiento [2,5]. Este artículo está organizado de la siguiente forma:

Primero se muestra las especificaciones y características del vehículo eléctrico y el modelo que es usado para este estudio. Después se describirán el Sistema difuso usado para la gestión de potencia. Se muestran los resultados experimentales de la solución propuesta y analiza la limitación dinámica de la corriente de acuerdo con el estado de carga del banco de baterías. Finalmente, las conclusiones se harán en la última sección.

\section{Trabajos relacionados}

En México, diversas universidades han desarrollado EV's que cumplen estándares internacionales, la Facultad de Ingeniería de la UNAM recientemente construyó el auto "Kalani" el cual es un vehículo monoplaza tripoide, con una estructura de acero y fibra de vidrio. Tiene un peso de 50 kilos y mide 120 centímetros de ancho por 220 


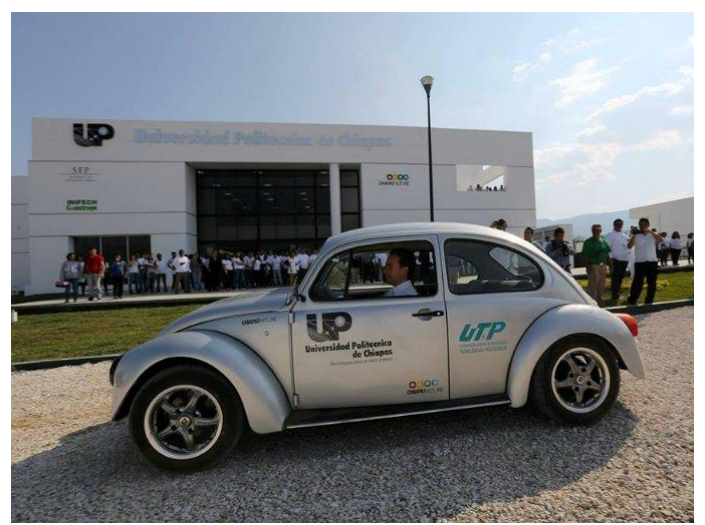

Fig. 1. Carro eléctrico UPChis01.

centímetros de largo y 80 centímetros de altura. Cuenta también con celdas de litio de 1000 watts y una eficiencia de 14 kilómetros de distancia en una sola carga.

Por otra parte, la Escuela Superior de Ingeniería Mecánica y Eléctrica (ESIME) del Instituto Politécnico Nacional (IPN) modificaron la estructura y diseño de un auto Volkswagen sedán para construir un carro con un motor de corriente directa que funciona con un rango de voltaje de 36 a 92 voltios, que es alimentado con un banco de seis baterías de ácido plomo de ciclo profundo de $8 \mathrm{~V}$ cada una. El Centro de Investigación en Mecatrónica Automotriz del Instituto Tecnológico de Estudios Superiores de Monterrey (ITESM), campus Toluca, actualmente se encuentran desarrollando un vehículo eléctrico para ser comercializado por empresas para la distribución de sus productos dentro de ciudades, este proyecto está en coordinación con la Secretaría de Energía.

Para garantizar la utilización completa de la energía disponible de las baterías de un EV a diferentes velocidades, la mayoría de los esquemas utilizan un controlador PID para administrar la energía de las baterías. El controlador PID convencional requiere un poco de ajuste para obtener una respuesta rápida y dinámicamente aceptable. De nuevo, generalmente se implementa utilizando circuitos amplificadores operacionales cuyos parámetros se ajustan para un punto de operación basado en un modelo lineal por piezas del sistema no lineal. Estos circuitos se ven afectaos por el tiempo de uso y la temperatura, lo que causa la degradación del rendimiento del sistema.

En diversos artículos [2,5,10] se presentan controladores basados en sistemas de lógica difusa para controlar la potencia de salida de un inversor de ancho de pulso (PWM) utilizado en un esquema de conversión de energía de un motor eléctrico. El generador de inducción autoexcitado utilizado en esos motores tienen el problema inherente de fluctuaciones en la magnitud y frecuencia de su voltaje con los cambios en la velocidad. Para evitar ese inconveniente, la magnitud variable, el voltaje de frecuencia variable en los terminales del generador se rectifica y la potencia de CC se transfiere a la carga a través de un inversor PWM. El objetivo es rastrear y extraer la máxima potencia del sistema de energía y transferir esta potencia a la carga aislada local. 


\section{Modelado del carro eléctrico}

\subsection{Componentes del carro eléctrico}

Primeramente, se describe características generales y componentes del vehículo eléctrico. Vehículo VW originalmente de motor de combustión interna de 4 cilindros, modelo del año 2000 con una masa de $550 \mathrm{~kg}$. La descripción básica de los componentes del EV UPCis01 está descrito en la tabla 1.

Existen diferentes tipos de arquitectura [6] algunas posibilidades son: 1 a 4 máquinas eléctricas, Maquinas eléctricas AC o DC, con o sin caja de cambios, alto o bajo voltaje en baterías, una o 3 fases de carga. La arquitectura elegida es la mostrada en la Fig. 2.

\subsection{Análisis matemático}

Las fuerzas que el vehículo eléctrico debe superar son fuerzas debido a la gravedad, viento, resistencia a la rodadura y efecto inercial [3]. Dichas fuerzas se pueden observar en la Fig. 3.

La fuerza de tracción de un vehículo puede ser descrito por las dos ecuaciones siguientes:

$$
\begin{gathered}
F_{\text {traccion }}=\underbrace{M_{E V} \dot{v}_{E V}}_{\text {Finercia }}+M_{E V} g \sin (\alpha)+\operatorname{signo}\left(v_{E V}\right) \underbrace{\overbrace{M_{E V} g \cos (\alpha)}^{\text {Fnormal }} c_{r r}}_{\text {Ffricion }}+ \\
+\operatorname{signo}\left(v_{E V}+v_{\text {viento }}\right) \underbrace{\frac{1}{2} \rho_{\text {aire }} C_{\text {arrastre }} A\left(v_{E V}+v_{\text {viento }}\right)^{2}}_{\text {Fviento }}, \\
C_{\text {arrastre }}=0.01\left(1+\frac{3.6}{100} v_{E V}\right) .
\end{gathered}
$$

Las descripciones de cada una de las variables en el modelo se muestran en la tabla 2.

\subsection{Baterías eléctricas}

Estos son rangos generales de voltaje para baterías de 6 celdas de plomo y ácido:

1. Circuito abierto (inactivo) a plena carga: $12,6 \mathrm{~V} \sim 12,8 \mathrm{~V}(2,10 \sim 2,13 \mathrm{~V}$ por celda).

2. Circuito abierto a plena descarga: $11,8 \mathrm{~V} \sim 12,0 \mathrm{~V}$.

3. Cargado a plena descarga: $10,5 \mathrm{~V}$. 
Tabla 1. Descripción de los componentes del EV.

\begin{tabular}{cl}
\hline Cantidad & \multicolumn{1}{c}{ Descripción } \\
\hline 1 & Motor eléctrico DC \\
\hline 1 & Controlador de motor \\
\hline 12 & Baterías 6 VCD \\
\hline 1 & Transmisión manual 5 vel. y 1 reversa \\
\hline 4 & Llantas rin 16 \\
\hline
\end{tabular}

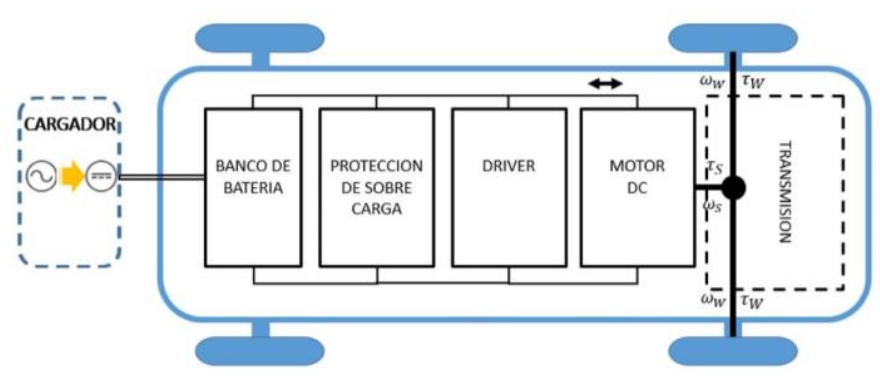

Fig. 2. Principales componentes del EV.

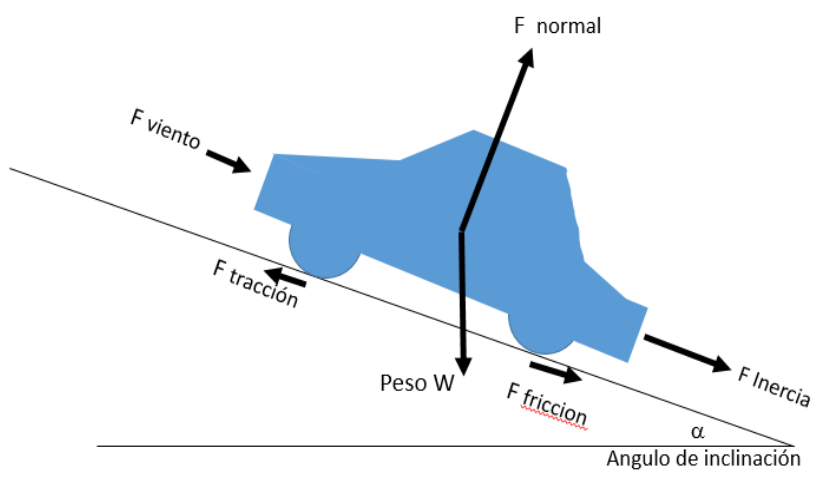

Fig. 3. Análisis de fuerzas del EV.

4. Carga continua de preservación (flotación): $13,4 \mathrm{~V}$ para electrolito de gel; 13,5 V para AGM (absorbed glass mat), y 13,8 V para celdas de electrolito fluido común.

5. Todos los voltajes están referenciados a $20^{\circ} \mathrm{C}$, y deben ajustarse $-0,022 \mathrm{~V} /{ }^{\circ} \mathrm{C}$ por cambios en la temperatura.

6. Las recomendaciones sobre el voltaje de flotación varían, de acuerdo con las recomendaciones del fabricante.

7. Una tensión de flotación precisa $( \pm 0,05 \mathrm{~V})$ es crítica respecto a la longevidad; muy baja (sulfatación) es casi tan mala como muy alta (corrosión y pérdida de electrolito) 
Ismael Osuna Galán, Yolanda Pérez Pimente, Juan Villegas Cortez, Carlos Avilés Cruz.

Tabla 2. Descripción de parámetros del modelo del EV.

\begin{tabular}{|c|c|c|}
\hline PARÁMETRO & DESCRIPCIÓN & UNIDADES \\
\hline$F_{\text {traccion }}$ & Fuerza de tracción & $\mathrm{N}$ \\
\hline$F_{\text {inercia }}$ & Fuerza de inercia & $\mathrm{N}$ \\
\hline$F_{\text {friccion }}$ & Fuerza de fricción & $\mathrm{N}$ \\
\hline$W$ & Peso de EV & $\mathrm{N}$ \\
\hline$F_{\text {normal }}$ & Fuerza normal & $\mathrm{N}$ \\
\hline$F_{\text {viento }}$ & $\begin{array}{l}\begin{array}{l}\text { Fuerza de resistencia } \\
\text { debida al viento }\end{array} \\
\end{array}$ & $\mathrm{N}$ \\
\hline$\alpha$ & Angulo de la superficie & Radianes \\
\hline$M_{E V}$ & Masa de EV & $\mathrm{Kg}$ \\
\hline$v_{E V}$ & Velocidad de EV & $m / s$ \\
\hline$\dot{v}_{E V}$ & Aceleración de EV & $m / s^{2}$ \\
\hline$\rho_{\text {aire }}$ & $\begin{array}{c}\text { Densidad del aire a } 30^{\circ} \mathrm{C} \\
1.650\end{array}$ & $\mathrm{~kg} / \mathrm{m}^{3}$ \\
\hline$A$ & Área frontal & $m^{2}$ \\
\hline$c_{r r}$ & $\begin{array}{l}\text { Resistencia del neumático al } \\
\text { rodamiento }\end{array}$ & \\
\hline$C_{\text {arrastre }}$ & $\begin{array}{lll}\begin{array}{l}\text { Coeficiente } \\
\text { aerodinámico }\end{array} & \text { fricción } \\
\end{array}$ & \\
\hline$v_{\text {aire }}$ & Velocidad del viento & $\mathrm{m} / \mathrm{s}$ \\
\hline
\end{tabular}

8. Carga típica (diaria): 14,2 V a 14,5 V (dependiendo de las recomendaciones del fabricante).

9. Carga de ecualización (baterías de electrolito fluido): 15 V para no más de 2 horas. La temperatura de la batería debe controlarse.

10. Después de plena carga la tensión de terminales caerá rápidamente a 13,2 V y luego lentamente a $12,6 \mathrm{~V}$.

\section{Construcción del sistema difuso}

En esta sección se describe el desarrollo del sistema difuso. En [4] se creó una prueba experimental para obtener la energía eléctrica consumida por un coche eléctrico de juguete, utilizada como prueba experimental para los controladores propuestos. El objetivo principal de este trabajo fue aplicar un controlador de lógica difusa para verificar si el controlador mejora el consumo de energía del EV. Siguiendo esas ideas, 
se desarrolló un sistema de inferencia difuso tipo Mamdami. Se usó el software LabVIEW para implementar ese sistema de forma embebida en un controlador CompactRIO de la empresa National Instruments.

Es un controlador tipo MISO (entradas múltiples y salida única), que tiene como entradas: pendiente de camino, profundidad de descarga y velocidad de conducción; como salida: la nueva velocidad del vehículo.

\subsection{Conjuntos difusos}

En este caso el sistema difuso es un administrador de división de potencia y controla todos los sistemas incorporados en el vehículo. Mediante una red de controlador CAN (acrónimo del inglés Controller Area Network) que es un protocolo de comunicaciones basado en una topología bus para la transmisión de mensajes en entornos distribuidos, dicha red es utilizada para comunicar todos los dispositivos.

Se muestran las variables de entrada y salida programadas así como las variables lingüísticas. Algunas razones para describir por qué usar funciones de pertenencia trapezoidal y triangular se mencionan en [9], donde se hace una comparación entre distintas funciones de pertenencia, señalando las mejores situaciones para cada uso. Los tres casos especiales para la opción Trapezoidal se destacan a continuación:

1. Construcción: se refiere a los métodos para obtener las funciones de membresía. Generalmente, hay dos métodos para crear una función de pertenencia: Model-Driven se necesita un modelo matemático para describir la planta y utilizan técnicas de optimización para ajustar los parámetros. Es más fácil de modelar variables si se hace una linealización como es el caso para controlar el combustible requerido por el controlador de potencia.

2. Monotonicidad: Describe cómo un sistema conserva la estructura original durante un proceso. Una función monótona se expresa como una expresión matemática que no cambia el orden dado. Una función trapezoidal funciona mejor que Gaussiana y otros;

3. Costo computacional: en tareas de control en tiempo real, se prefiere un algoritmo de bajo costo, en otras palabras, el controlador lleva a cabo un proceso más rápido. Al hacer un sistema de Mamdani con 50 reglas o menos que eso una función del tipo Gaussiano es mejor. Sin embargo, con el caso de este estudio, se utilizan más de 100 reglas, una inferencia con trapecios y triángulos se realiza más rápido.

\subsection{Reglas difusas}

Las tareas del controlador de disminuir la velocidad EV para reducir el consumo de energía del vehículo. En primer lugar, la pendiente de las entradas, derivadas del gradiente de la carretera, profundidad de descarga, calculada a partir del modelado del EV y la velocidad del vehículo.

Se usaron 224 reglas, el controlador difuso actúa en situaciones donde la profundidad de descarga es mayor al $70 \%$ y se aplica directamente en el rendimiento del vehículo, el sistema disminuye la velocidad para proteger la energía de la batería al reducir en el 


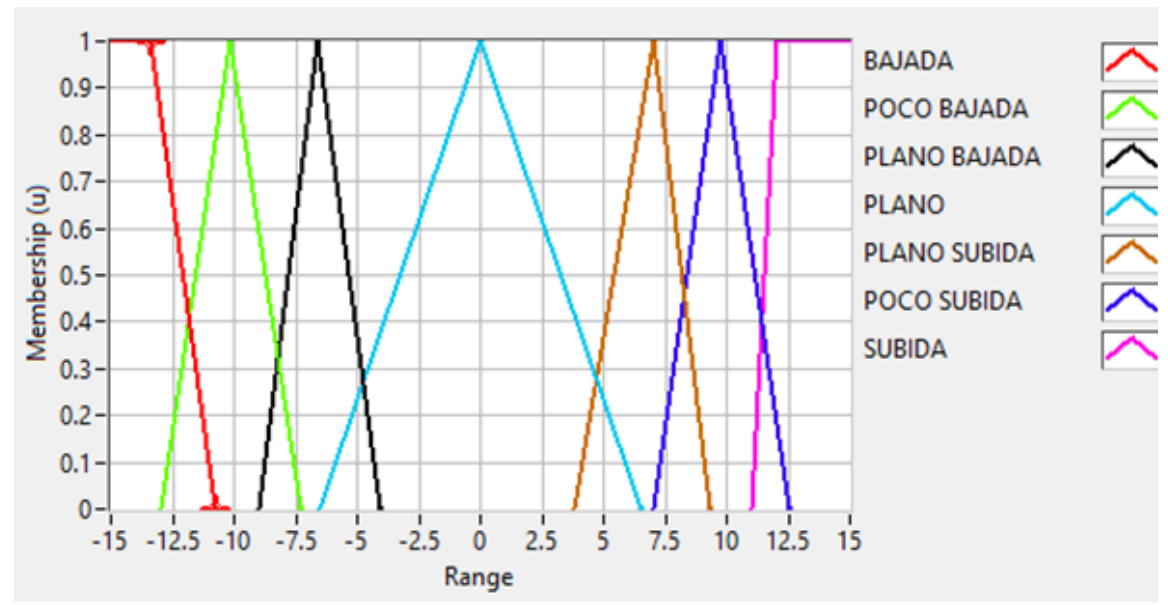

Fig. 4. Descripción de la variable de entrada PENDIENTE.

consumo de energía eléctrica, ya que es directamente proporcional a la fuerza de tracción del EV. Este controlador funciona en diferentes entornos (pendiente llana de la carretera, escenarios ascendentes y descendentes).

Por ejemplo, una de estas reglas es la siguiente:

"PENDIENTE $=$ PLANO and DESCARGA $=$ ECO MODE y VELOCIDAD = ALTA then VELOCIDAD CORREGIDA = MEDIA BAJA".

En este caso, el controlador difuso reduce la velocidad (VELOCIDAD CORREGIDA) al detectar una descarga de las baterías superior al 70\% (conjunto ECO MODE) por lo que la velocidad solicitada por el usuario es ignorada por las condiciones de carretera (conjunto PLANO).

\section{Pruebas experimentales}

Un ciclo de conducción se compone de micro-viajes y tiene un período de 5 a 40 minutos. Esta duración debe contener suficientes micro-viajes que reflejen el comportamiento de conducción en el mundo real.

Los ciclos de manejo pueden ser de laboratorio o reales [4]. Los siguientes son algunos parámetros básicos del ciclo fue realizado por un tramo de la nueva carretera Tuxtla Gutíerrez - Suchiapa-Villaflores; Duración: 499 s; Distancia recorrida: 5.4 km; Velocidad media: $60.2 \mathrm{~km} / \mathrm{h}$; Velocidad máxima: $85.5 \mathrm{~km} / \mathrm{h}$. La velocidad del ciclo de conducción y la velocidad controlada se expresan en la Fig. 8.

En cualquier caso, es importante obtener variables principales como velocidad, aceleración, distancia y pendiente de la ruta. Cuando se habla sobre el desarrollo del ciclo de manejo, tres pasos son importantes: selección de ruta, recolección de datos y construcción de ciclo. La selección de ruta implica seleccionar el curso para describir el ciclo. Consiste en determinar si la ruta es una autopista con velocidad constante, vías arteriales o conducción urbana, por ejemplo. 


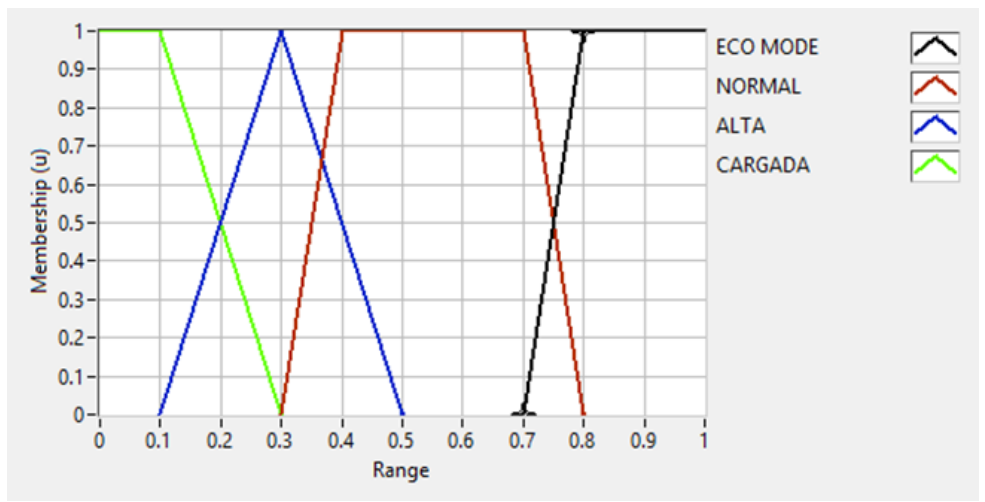

Fig. 5. Descripción de la variable de entrada DESCARGA.

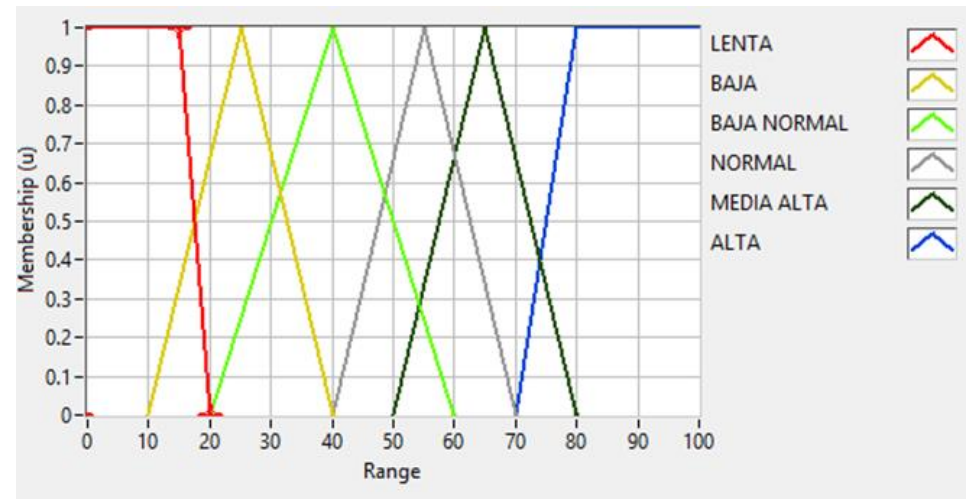

Fig. 6. Descripción de la variable de entrada VELOCIDAD.

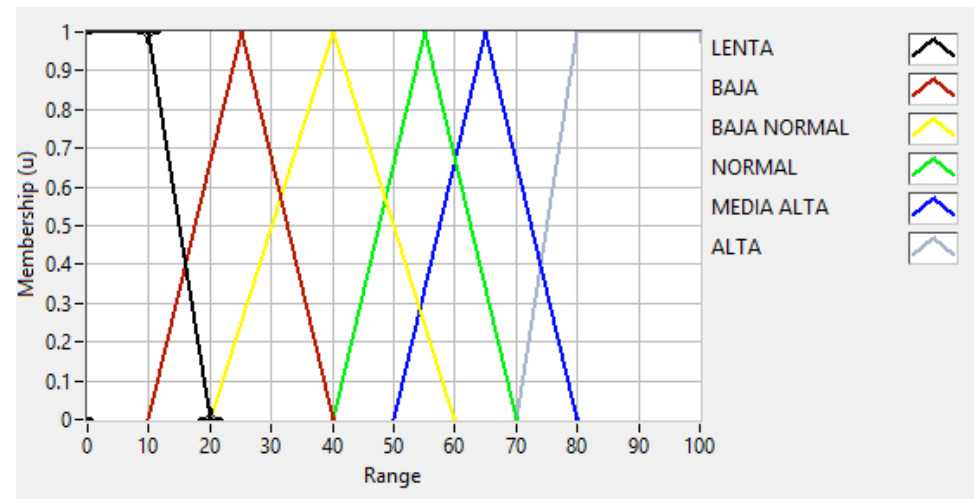

Fig. 7. Descripción de la variable de salida VELOCIDAD CORREGIDA.

La recopilación de datos es la capacidad de recopilar los parámetros, los datos con los sensores adecuados para describir el ciclo de conducción. Finalmente, para la 
Ismael Osuna Galán, Yolanda Pérez Pimente, Juan Villegas Cortez, Carlos Avilés Cruz.

Tabla 3. Valores promedio de pérdida de potencia durante un ciclo de viaje.

\begin{tabular}{ccc}
\hline PARÁMETRO & RESULTADO EV & MEJORA \\
\hline $\begin{array}{c}\text { Pérdida de potencia sin } \\
\text { controlador }\end{array}$ & $1479 \mathrm{~W}$ & \\
\hline $\begin{array}{c}\text { Pérdida de potencia con } \\
\text { controlador }\end{array}$ & $1135 \mathrm{~W}$ & $23.25 \%$ \\
\hline
\end{tabular}

Tabla 4. Valores promedio de corriuete durante un ciclo de viaje.

\begin{tabular}{ccc}
\hline PARÁMETRO & RESULTADO EV & MEJORA \\
\hline $\begin{array}{c}\text { Banco de baterías sin } \\
\text { controlador } \\
\text { controlador }\end{array}$ & $34.95 \mathrm{~A}$ \\
\hline & $26.44 \mathrm{~A}$ \\
\hline VELOCIDAD SIN CONTROLADOR & $24.34 \%$ \\
\hline
\end{tabular}

Fig. 8. Velocidades del EV durante un mini viaje.

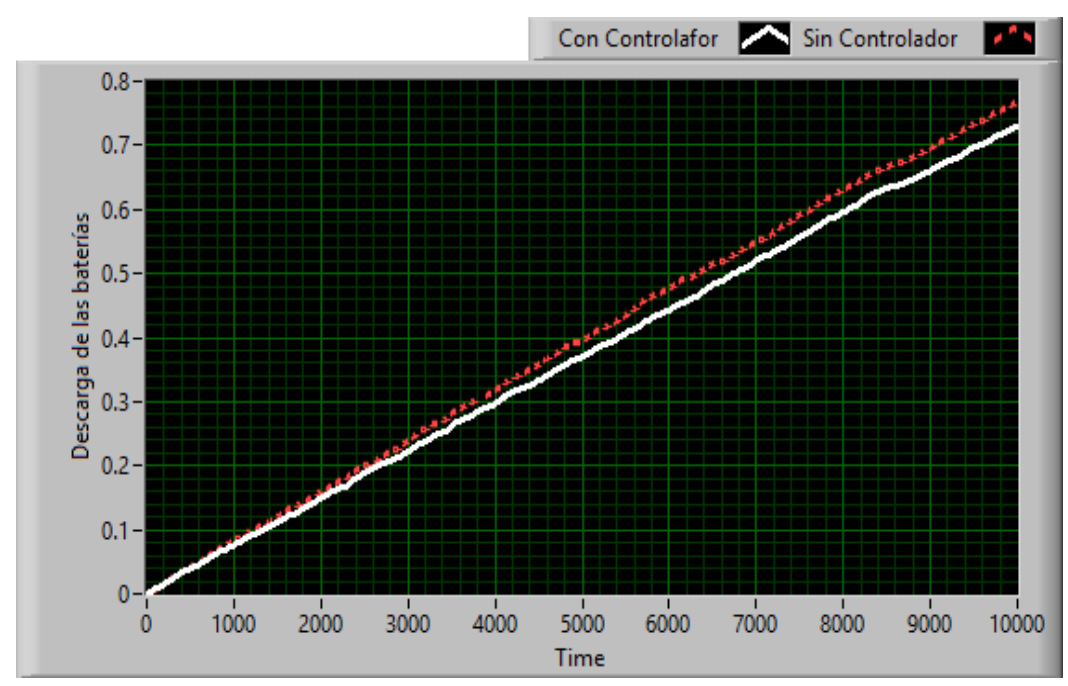

Fig. 9. Tiempo de descarga del banco de baterías del EV.

construcción del ciclo se dividen todos los datos en micro viajes y se construye una función de dominio del tiempo de la velocidad del vehículo. 
En las siguientes tablas, se muestran las pérdidas de potencia del motor eléctrico. Con esas pérdidas, se produjo la pérdida de calor en la batería. Los valores medios obtenidos en los resultados experimentales muestran una mejora general del $23.7 \%$ con la aplicación del sistema difuso. La tabla 3 y tabla 4 dan muestra de la mejora en el rendimiento del banco de baterías.

En la Fig. 9, se ilustra la profundidad de la descarga en función del tiempo. La mayor autonomía del vehículo es visible cuando el ciclo con el controlador tiene un intervalo de tiempo más largo para drenar toda la batería.

El ciclo de conducción se repite hasta que ciclo de descarga alcanza el 95\%, que es un estado de batería vacía para analizar la distancia adicional dada por el sistema desarrollado.

\section{Conclusiones}

Las pruebas experimentales muestran que con el controlador integrado en el EV se obtuvieron los resultados deseados. En todos ellos, se generaron valores más bajos para todos los parámetros analizados, lo que demuestra la efectividad de la implementación del sistema. El consumo de energía eléctrica tuvo una reducción en todas las pruebas, dando integridad y seguridad al banco de baterías [1].

El tiempo más largo alcanzado por el vehículo es un resultado necesario, evitando la batería vacía y dando la oportunidad al conductor de encontrar una próxima estación de recarga otorgando una pequeña ganancia en la distancia de viaje.

Será necesario verificar si el comportamiento para la autopista tiene el mismo comportamiento el sistema que en un medio urbano.

Es probable que la velocidad se vea reducida de manera natural por las condiciones de tráfico y límites de velocidad que ocurren en una ciudad.

Es de suponer que en la mayoría de los escenarios de autopista ocurran mayores velocidades por lo que el EV puede ser una solución viable para la comunicación entre pequeñas poblaciones cercanas a la ciudad. Por lo tanto, para mejorar el análisis del sistema y realizar diferentes investigaciones sobre los temas adoptados en el proyecto.

Las sugerencias para futuros estudios son en el sentido de mejorar el modelo del motor eléctrico usando un modelo de batería Li-Ion o la aplicación nuevos tipos de controladores como PID difuso o redes neuronales.

\section{Referencias}

1. García-López, M., et al.: Análisis de pérdidas en semiconductores de potencia generadas por controladores difusos de velocidad en motores de CD sin escobillas. Research in Computing Science, 135, pp. 115-127 (2017)

2. Hanane, H., Ghouili, J., Cheriti, A.: A real time fuzzy logic power manage-ment strategy for a fuel cell vehicle. Energy conversion and Management 80, pp. 63-70 (2014)

3. Hibbeler, R.: Engineering Mechanics Dynamics. [S.1.]: Upper Saddle River, NJ: Pearson Prentice Hall (2016)

4. Howey, D.A., et al.: Comparative measurements of the energy consumption of 51 electric, hybrid and internal combustion engine vehicles. Transportation Research Part D: Transport and Environment, 16, pp. 459-464 (2011) 
5. Kandi, M., Soleymani, M., Ghadimi, A.A.: Designing an optimal fuzzy controller for a fuel cell vehicle considering driving patterns. Scientia Iranica. Transaction B, Mechanical Engineering, 23, pp. 218-235 (2016)

6. Larminie, J., Lowry, J.: Electric vehicle technology explained. John Wiley \& Sons (2012)

7. Maia, R., et al.: Electric vehicle simulator for energy consumption studies in electric mobility systems. In: Integrated and Sustainable Transportation System (FISTS), 2011 IEEE Forum on. IEEE (2011)

8. Tariq, M., Kolhe, M., Doyle, A.: Electric Vehicles: Prospects and Challenges. Elsevier (2017)

9. Dongrui, W.: Twelve considerations in choosing between Gaussian and trapezoidal membership functions in interval type-2 fuzzy logic controllers. In: International Conference on Fuzzy Systems (FUZZ-IEEE) (2012)

10. Al-Jazaeri, A.O., Samaranayake, L., Longo, S., Auger, D.J.: Fuzzy logic control for energy saving in autonomous electric vehicles. In: The IEEE International Electric Vehicle Conference (IEVC), pp. 1-6 (2014) 